\title{
E Rapid Treatment of Acute Ischemic Stroke Using a Computed Tomography-Based Reperfusion Protocol: The Reality of a Local Community Hospital with Limited Resources
}

Yui Mano, ${ }^{1}$ Ichiro Suzuki, ${ }^{2}$ Syuichi Ishikawa, ${ }^{1}$ Masahito Katsuki, ${ }^{3}$ Ryutaro Suzuki, ${ }^{1}$ Takaki Ichikawa, ${ }^{4}$ Yuji Kato, ${ }^{4}$ Ryosuke Sato, ${ }^{4}$ Masaya Toyoshima, ${ }^{4}$ Kazuhiro Kato, ${ }^{4}$ Koichi Narikawa, ${ }^{4}$ Takanori Oikawa, ${ }^{4}$ and Teiji Tominaga ${ }^{3}$

Objectives: In patients with acute ischemic stroke (AIS), prognosis strongly depends on the onset-to-recanalization time. The Ishinomaki protocol for rapid recanalization has been used since October 2017. This protocol determines the indication for reperfusion therapy based on computed tomography (CT)/three-dimensional CT angiography (3DCTA) findings and intends to reduce the onset-to-recanalization time. We aimed to compare the outcomes before and after protocol introduction. Methods: Our hospital is the only thrombectomy-capable center in Ishinomaki, Tome, and Kesennuma medical area. Before protocol introduction (April 2014-June 2016), both CT and magnetic resonance imaging were performed to determine the indications for intravenous (IV) recombinant tissue-plasminogen activator (rt-PA) or mechanical thrombectomy within 6 hours of disease onset. However, after protocol introduction (from October 2017), plain CT and 3DCTA were used. We collected data on patients who underwent mechanical thrombectomy and/or IV rt-PA before $(n=13)$ and after $(n=34)$ the protocol introduction. The required time from onset to door (OTD), door to needle (DTN), needle to puncture (NTP), puncture to recanalization (PTR), and door to recanalization (DTR) were compared before and after protocol introduction. Furthermore, thrombolysis in cerebral infarction $(\mathrm{TICl})$ grades and modified Rankin scale (mRS) scores at discharge were compared.

Results: The outcomes before and after protocol introduction were as follows: OTD: $105 \pm 73.8$ (mean \pm standard deviation) vs. $120 \pm 68.1 \mathrm{~min}$ ( $p=0.376$, Mann-Whitney $U$ test); DTN: $62.9 \pm 15.9$ vs. $41 \pm 17 \min (p<0.01)$; NTP: $112 \pm 69.8$ vs. $39.9 \pm 33.7 \min (p<0.01)$; PTR: $87.9 \pm 45.4$ vs. $52.5 \pm 27.9 \min (p<0.01)$; and DTR, $230 \pm 69.9$ vs. $110 \pm 40.3 \mathrm{~min}(p<0.0001)$. Before and after protocol introduction, the proportion of patients with $\mathrm{TICl}$ grade $2 \mathrm{~b}-3, \mathrm{mRS}$ score of $0-2$ at discharge, and mRS score of $5-6$ were $54 \%$ vs. $50 \%$ ( $p=0.815$, Fisher's exact test), $23 \%$ vs. $21 \%$ $(p=0.854)$, and $15 \%$ vs. $50 \%(p=0.046)$, respectively.

Conclusion: The Ishinomaki protocol reduced the mean DTR time by $120 \mathrm{~min}$. The reduction in treatment time was due to the change in CT-based recanalization and collaboration with emergency physicians and paramedics. There was no increase in good outcomes, but there was a significant increase in poor outcomes at discharge. Patients who could not be salvaged were indicated for reperfusion therapy as CT and 3DCTA cannot detect the ischemic core.

Keywords > acute ischemic stroke, thrombectomy, recombinant tissue plasminogen activator, recanalization

\footnotetext{
${ }^{1}$ Department of Neurosurgery, Japanese Red Cross Ishinomaki Hospital, Ishinomaki, Miyagi, Japan

${ }^{2}$ Department of Neurosurgery, Hachinohe City Hospital, Hachinohe, Aomori, Japan

${ }^{3}$ Department of Neurosurgery, Tohoku University Graduate School of Medicine, Sendai, Miyagi, Japan

${ }^{4}$ Department of Neurology, Japanese Red Cross Ishinomaki Hospital, Ishinomaki, Miyagi, Japan
}

Received: September 28, 2020; Accepted: November 8, 2020
Corresponding author: Yui Mano, Department of Neurosurgery, Ohara General Hospital, 6-1, Uwamachi, Fukushima, Fukushima 960-8611, Japan

Email: yui.manyo@gmail.com

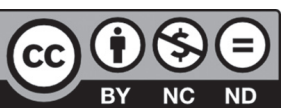

This work is licensed under a Creative Commons Attribution-NonCommercialNoDerivatives International License. 


\section{Introduction}

Reperfusion therapy in acute ischemic stroke (AIS) has evolved dramatically in the past 20 years. Initially, only intravenous (IV) alteplase (recombinant tissue-plasminogen activator [rt-PA]) therapy was indicated for patients who experienced AIS within 3 hours. According to the accumulating evidence, rt-PA can be administered to patients within 4.5 hours of "discovery,", thrombectomy can be performed within 24 hours of onset in a patient with large vessel occlusion and a mismatch between the penumbra and ischemic core volume..$^{3-5)}$

The rapid expansion of the therapeutic time window and the increasing level of evidence have led to the adoption of thrombectomy for the treatment of eligible patients without delay, which increased the physical and mental burden of the stroke team in regional hospitals.

The time required for onset to reperfusion has a significant impact on patient prognosis, ${ }^{6,7)}$ and the inter-hospital transfer of patients may lead to poor outcomes. ${ }^{8,9)}$ Therefore, a medical system that enables the completion of IV rt-PA followed by mechanical thrombectomy needs to be established in each medical region. However, the current number of doctors performing thrombectomy in rural areas is insufficient.

We started mechanical thrombectomy in April 2014. From 2014 to 2016, the indications for reperfusion therapy were evaluated on a case-by-case basis after conducting magnetic resonance imaging (MRI). After 2017, when the evidence was established, we created a CT-based reperfusion protocol for AIS therapy (Ishinomaki protocol), which is aimed at shortening the time from the arrival of a patient until the administration of treatment. In this study, we compared the pre- and post-protocol phases based on the required time for reperfusion therapy and treatment outcomes that include effective recanalization rate and modified Rankin scale (mRS) score at discharge.

\section{Materials and Methods}

The Japanese Red Cross Ishinomaki Hospital is the main hospital in Ishinomaki, Tome, and Kesennuma medical area where the population consists of approximately 350,000 people. This medical area has three centers that offer 24-hour IV rt-PA, and our hospital (Japanese Red Cross Ishinomaki Hospital) is the only medical institution that performs thrombectomy.

During the pre-protocol phase (2014-2016), three or four neurosurgeons, including one neuroendovascular surgeon and one neurologist, treated the patients with stroke. Since October 2017 (post-protocol phase), the stroke team comprised two neurosurgeons, including one neuroendovascular surgeon, and four neurologists.

Since October 2017, our emergency department physicians, junior and senior residents, nurses, clinical engineering technologists, and radiology technicians have learned about the Ishinomaki protocol. The indications for this protocol are as follows: (i) detection within 6 hours of onset or last known well time, (ii) one or more positive items on the Cincinnati Prehospital Stroke Scale, ${ }^{10)}$ and (iii) pre-morbid mRS score of 0-2 (independence in activities of daily living) (Fig. 1). For patients in whom stroke was detected between 6 and 24 hours of onset, criteria (ii) and (iii) were the common indications for the Ishinomaki protocol. The Diffusion-Weighted Imaging-Alberta Stroke Program Early Computed Tomography Score (DWI-ASPECTS) should be determined by performing MRI in accordance with the guidelines before determining the indication for thrombectomy. ${ }^{5)}$ Prehospital education on the Immediate Stroke Life Support/Prehospital Stroke Life Support course was held. We informed the paramedics in this medical area and asked them to take the patient to our hospital. In cases of early presenting stroke, which means the stroke occurred within 6 hours or is determined based on the patient's last known well time, emergency requests are made for patients who met all of the above criteria; the emergency physician should call a neurosurgeon or neurologist on duty before the patient's arrival. The neurosurgeon or neurologist on duty would examine the patient upon arrival. In the pre-protocol phase, treatment decisions for AIS were based on the patient's MRI findings. However, in the post-protocol phase, the performance of an MRI scan was omitted, and plain CT and three-dimensional CT angiography (3DCTA) were conducted simultaneously in the CT room adjacent to the emergency room. Immediately after the performance of plain CT and 3DCTA, neurosurgeons and neurologists formulated the treatment plan, and one of the following treatment options was selected immediately: (i) IV rt-PA plus mechanical thrombectomy, (ii) IV rt-PA alone, (iii) IV rt-PA skipping and mechanical thrombectomy, and (iv) conservative therapy (Fig. 1). The paramedical staff are immediately informed of the treatment plan in the emergency CT room and performed their individual tasks. In particular, clinical engineering technologists immediately set up the devices, such as priming the balloon guide catheter and some perfusion lines, and provided intraoperative technical support.

Either TrevoXP $4 \mathrm{~mm} \times 20 \mathrm{~mm}$ (Stryker, Kalamazoo, MI, USA) or Penumbra 5 MAX (Penumbra, Alameda, CA, 
Flowchart of reperfusion for acute ischemic stroke (onset from $<6 \mathrm{hrs}$ )

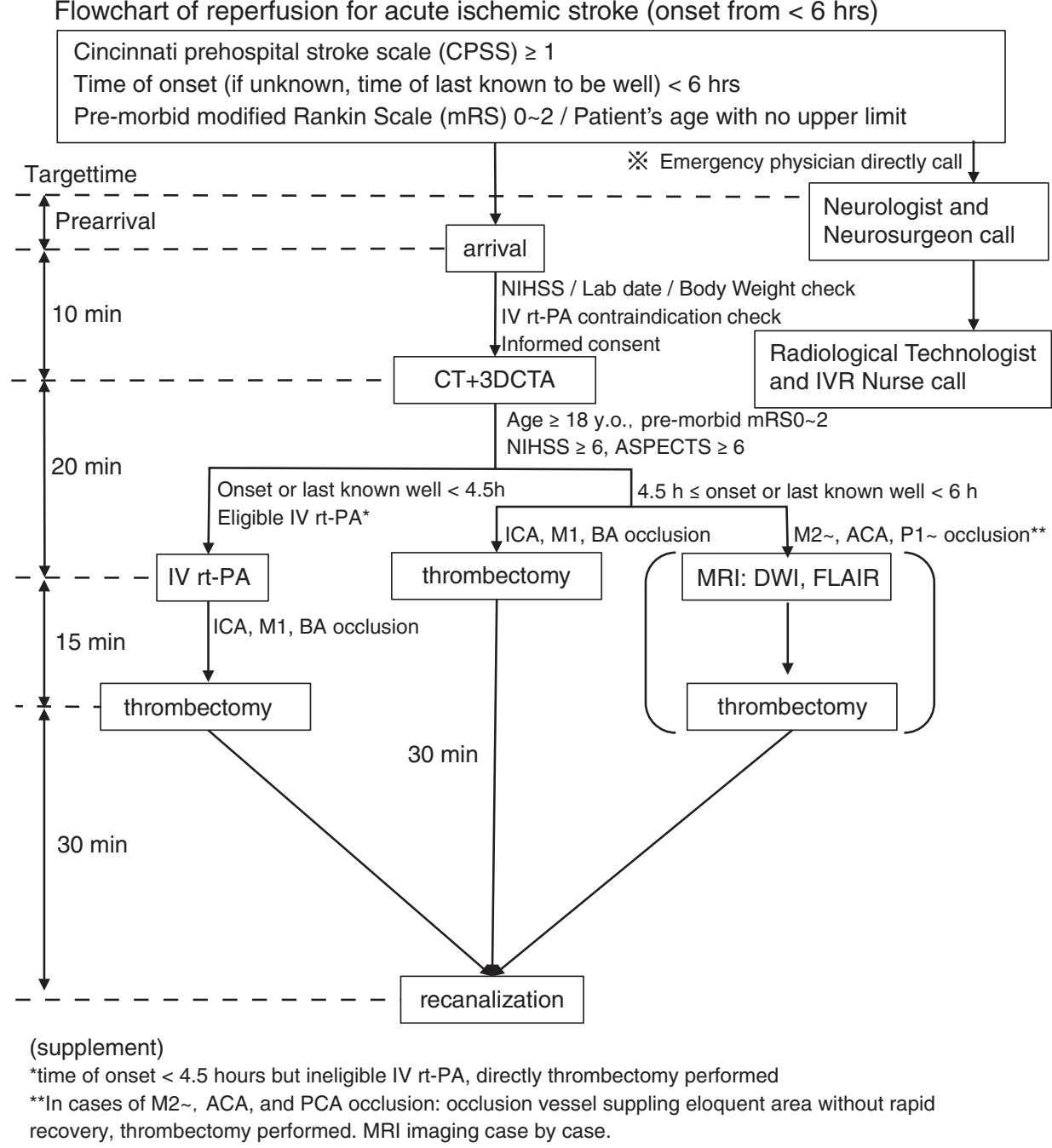

Fig. 1 Flowchart of reperfusion for AIS protocol (Ishinomaki protocol). In case of onset less than 6 hours. Patients who are positive on the Cincinnati Prehospital Stroke Scale for more than one item and have a premorbid mRS score of less than 2 accept the request from EMS without delay. Emergency physicians call the neurologist and the neurosurgeon on duty before EMS arrival. The neurologist and neurosurgeon examine the patients upon arrival and obtain an informed consent in collaboration with the emergency physician and junior residents. Blood sampling, vital signs, and National Institute of Health Stroke Scale score are assessed, and then CT is performed; if no hemorrhage is noted, continuous monitoring with 3DCTA is performed. CT-ASPECTS and occluded vessel were determined by performing CT and 3DCTA, and the need for rt-PA and/or mechanical thrombectomy is determined as soon as possible. 3DCTA: three-dimensional computed tomography angiography; AIS: acute ischemic stroke; CT: computed tomography; EMS: emergency medical service; mRS: modified Rankin scale; rt-PA: recombinant tissue-plasminogen activator

USA) was used as the thrombectomy device based on the surgeon's discretion and a balloon-guiding catheter was routinely used during the pre-protocol phase (2014-2016). In the post-protocol phase (2017-2020), the device selection was standardized according to the occluded vessel. For anterior circulation, a 9-Fr Cello (Medtronic, Minneapolis, MN, USA) was used as a balloon-guiding catheter. A direct aspiration first pass technique (ADAPT) ${ }^{11)}$ was performed using a by ACE 68 catheter (Penumbra), which was inserted from the cervical internal carotid artery (ICA) into the ICA genu. From the ICA genu to the M1 proximal part, the Solitaire platinum $6 \mathrm{~mm} \times 40 \mathrm{~mm}$ (Medtronic) was used. The Trevo XP $4 \mathrm{~mm} \times 20 \mathrm{~mm}$ was used from the M1 proximal to the M1-M2 bifurcation; beyond M2, the Trevo XP $3 \mathrm{~mm} \times 20 \mathrm{~mm}$ was used. For posterior circulation, a 6-Fr Fubuki with a dilator (ASAHI INTECC, Aichi, Japan) was used as a guiding catheter, and ADAPT with ACE 68 was the first-line treatment.

We extracted 13 cases from April 2014 to October 2016 (pre-protocol group) and 34 cases from October 2017 to 
Table 1 Patients' baseline demographic and clinical characteristics

\begin{tabular}{|c|c|c|c|}
\hline & $\begin{array}{c}\text { Pre-protocol } \\
2014 / 4 / 1-2016 / 6 / 30\end{array}$ & $\begin{array}{c}\text { Post-protocol } \\
2017 / 10 / 1-2020 / 3 / 31\end{array}$ & $p$ value* \\
\hline Number of patients & 13 & 34 & \\
\hline Mela: female & $7: 6$ & $17: 17$ & 0.813 \\
\hline Age (mean $\pm \mathrm{SD})$ & $77(40-87)$ & $79.5(57-93)$ & 0.124 \\
\hline \multicolumn{4}{|l|}{ Stroke type (\%) } \\
\hline Athrosclerotic & $2(15 \%)$ & $4(12 \%)$ & 0.739 \\
\hline Cardiogenic & $9(70 \%)$ & $23(68 \%)$ & 0.917 \\
\hline ESUS & $2(15 \%)$ & $7(20 \%)$ & 0.688 \\
\hline \multicolumn{4}{|l|}{ Occluded vessel (\%) } \\
\hline ICA & $3(23 \%)$ & $15(44 \%)$ & 0.315 \\
\hline M1 & $8(61 \%)$ & $10(29 \%)$ & 0.054 \\
\hline M2- & $1(8 \%)$ & $4(12 \%)$ & 0.689 \\
\hline BA & $1(8 \%)$ & $5(15 \%)$ & 0.519 \\
\hline Median CT-ASPECTS (min-max) & $8(6-10)$ & $8(6-10)$ & 0.525 \\
\hline Median ASPECTS-DWI (min-max) & $8(5-11)$ & - & \\
\hline Median pre-mobid mRS (min-max) & $0(0-2)$ & $0(0-2)$ & 0.307 \\
\hline Median NIHSS at arrival (min-max) & $16(11-40)$ & $21.5(7-40)$ & 0.202 \\
\hline MRI acquisition pre thrombectomy & Yes & No & \\
\hline i.v. rt-PA (\%) & $9 / 13(69 \%)$ & 29/34 (85\%) & 0.237 \\
\hline Complication (\%) & $1(8 \%)$ & $5(15 \%)$ & 0.524 \\
\hline During thrombectomy & 0 & $4(12 \%)$ & 0.303 \\
\hline Hemorrhagic infarction & 0 & $1(3 \%)$ & 0.923 \\
\hline External decompression needed & $1(8 \%)$ & 0 & 0.086 \\
\hline
\end{tabular}

${ }^{*} p$ value was calculated using Mann-Whitney $U$ test for continuous or ordinal variables and chi-square test or Fisher's exact test for categorical variables. ASPECTS: Alberta Stroke Program Early CT score; BA: basilar artery; DWI: diffusion-weighted imaging; ESUS: embolic stroke of undetermined source; ICA: internal carotid aftery; M: middle cerebral artery; mRS:

modified Rankin scale; NIHSS: National Institute of Health Stroke Scale; rt-PA: recombinant tissue-plasminogen activator

March 2020 (post-protocol group); both groups had a stroke onset within 6 hours or their last known well time was determined. We compared the time required from the onset to door (OTD), door to needle (DTN: the time needed to start IV rt-PA), needle to groin puncture (NTP), door-togroin puncture (DTP), groin puncture to recanalization (PTR), and door to recanalization (DTR) as well as the recanalization rate and patient status at discharge. The degree of recanalization was assessed using thrombolysis in cerebral infarction (TICI) grade, ${ }^{12}$ ) with TICI grades $2 b$ and 3 representing effective recanalization, and the rate of recanalization was compared before and after the adoption of the protocol. Patient prognosis was assessed using the mRS score at discharge, ${ }^{13)}$ with scores $0-2$ indicating a good outcome and scores 5-6 indicating a poor outcome. The percentage of patients with mRS scores of 0-2 and
5-6 were compared between the pre- and post-protocol phases.

Statistical analysis was performed using IBM SPSS for Windows, Version 22.0 (IBM Corp, Armonk, NY, USA). The $\chi$-square test was carried out to analyze the male/ female ratio, infarct type, occluded vessel, and IV rt-PA status. Age, ASPECTS, National Institute of Health Stroke Scale score upon arrival, and pre-morbid mRS scores were analyzed using the Mann-Whitney U test (Table 1).

The Mann-Whitney U test was used to measure the time required for treatment (Table 2), while the Fisher's direct probability test was used to measure the effective recanalization rate of TICI grades $2 b-3$ and the proportion of patients with mRS scores of $0-2$ and 5-6 at discharge (Figs. 2 and 3), with the significance level set at 0.05 . 
Table 2 Required time from arrival, examination to treatment (mean $\pm S D$ )

\begin{tabular}{lccl} 
& $\begin{array}{c}\text { Pre-protocol } \\
2014 / 4 / 1-2016 / 6 / 30\end{array}$ & $\begin{array}{c}\text { Post-protocol } \\
2017 / 10 / 1-2020 / 12 / 31\end{array}$ & $p$ value ${ }^{\dagger}$ \\
OTD & $105 \pm 73.8$ & $120 \pm 68.1$ & 0.376 \\
DTN & $62.9 \pm 15.9$ & $41 \pm 17$ & $0.00584^{*}$ \\
NTP & $112 \pm 69.8$ & $39.9 \pm 33.7$ & $0.00149^{*}$ \\
DTP & $137 \pm 80.4$ & $57.4 \pm 24$ & $0.00242^{*}$ \\
PTR & $89.7 \pm 45.4$ & $52.5 \pm 27.9$ & $0.00917^{*}$ \\
DTR & $230 \pm 69.9$ & $110 \pm 40.3$ & $0.0000025^{*}$ \\
\hline${ }^{*} p<0.05 .{ }^{\dagger} p$ value was calculated using the Mann-Whitney U test. DTN: door to needle; \\
DTP: door to puncture; DTR: door to recanalization; NTP: needle to puncuture; OTD: \\
onset to door; PTR: puncture to recanalization
\end{tabular}

\section{Results}

The time required for each treatment step was compared between the pre- and post-protocol phases. The OTDs were $105 \pm 73.8$ minutes in the pre-protocol phase and 120 \pm 68.1 minutes in the post-protocol phase, which was not significantly different ( $p=0.376$, Mann-Whitney $\mathrm{U}$ test). However, before and after the introduction of the protocol, all other required time values were $62.9 \pm 15.9$ vs. $41 \pm 17$ minutes for DTN $(p<0.01), 112 \pm 69.8$ vs. $39.9 \pm 33.7$ minutes for NTP ( $p<0.005), 137 \pm 80.4$ vs. $57.4 \pm 24$ minutes for DTP $(p<0.005), 89.7 \pm 45.4$ vs. $52.5 \pm 27.9$ minutes for PTR $(p<0.01)$, and $230 \pm 69.9$ vs. $110 \pm 40.3$ minutes for DTR $(p<0.0001)$, respectively (Table 2). The time required for all treatment steps except OTD was significantly reduced after the introduction of the protocol.

The TICI $2 \mathrm{~b}-3$ rates were $54 \%$ ( $7 / 13$ patients) in the pre-protocol phase and $50 \%$ (17/34 patients) in the post-protocol phase; the difference was not significant ( $p=0.815$, Fisher's exact test) (Fig. 2).

The proportions of patients with mRS score of $0-2$ at discharge were $23 \%$ ( $3 / 13$ cases) in the pre-protocol phase and $21 \%$ (7/34 cases) in the post-protocol phase, but the difference was not significant ( $p=0.854$, Fisher's exact test). Meanwhile, the proportions of patients with $\mathrm{mRS}$ score of 5-6 at discharge were $15 \%$ (2/13 cases) in the pre-protocol phase and 50\% (17/34 cases) in the post-protocol phase, but the difference was significant ( $p=0.046$, Fisher's exact test) (Fig. 3). For patients who had TICI 2b-3 recanalization alone, the proportions of those with mRS score of $0-2$ at discharge were $37.5 \%$ ( $3 / 8$ cases) in the pre-protocol phase and $41.2 \%$ (7/17 cases) in the post-protocol phase; however, the difference was not significant ( $p=0.607$, Fisher's exact test).
One patient had an external decompression due to brain edema in the pre-protocol phase, while four patients had a subarachnoid hemorrhage (SAH) due to thrombectomy and one had hemorrhagic infarction in the post-protocol phase (Table 1). In the post-protocol phase, one patient died due to nocturnal paroxysmal hemoglobinuria with $\mathrm{SAH}$ without neurological deterioration after thrombectomy. The next day, the patient experienced sudden cardiac arrest and did not recover despite resuscitation. Hyperkalemia due to hemolysis likely led to the patient's cardiac arrest.

\section{Discussion}

One of the important factors that improved the patients' outcomes after AIS was the rapid achievement of TICI 2b-3 recanalization. ${ }^{7,14}$ The Ishinomaki protocol is designed to improve patient's prognosis by minimizing the time required to initiate IV rt-PA and/or mechanical thrombectomy by performing quick initial examinations and determination of indications for treatment based on plain $\mathrm{CT}$ and 3DCTA findings. The shortening of the time from the arrival of a patient until the administration of treatment was accomplished. We tried to improve the patient's outcomes by reducing the time required for treatment, but we failed to do so in our hospital.

Following the introduction of the protocol, DTN, DTP, and DTR were reduced by an average of 21.9, 79.6, and $120 \mathrm{~min}$, respectively (Table 2). The main reasons for the time reduction were as follows. First, emergency physicians had to call the neurologist and neurosurgeon on duty to notify them about the delivery of a patient who met the criteria (Fig. 1). This enabled the stroke treatment doctors (neurologist and neurosurgeon) to intervene immediately upon the patient's arrival. Second, in the pre-protocol 


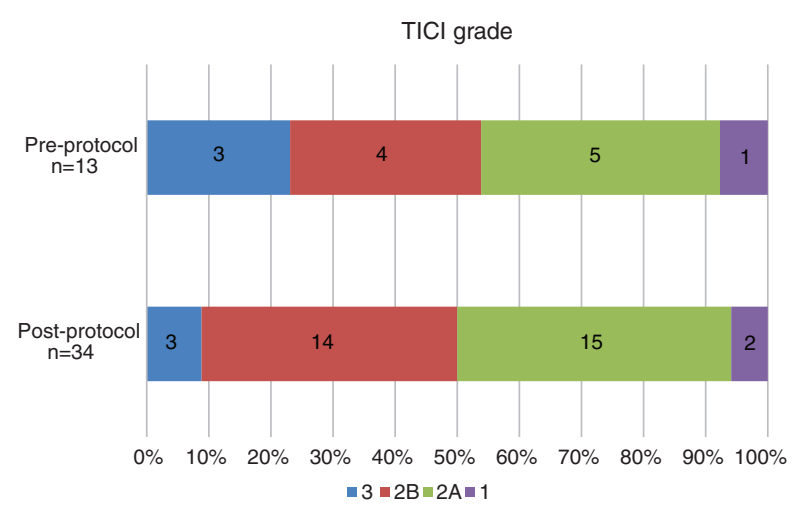

Fig. 2 The effective recanalization rates of $\mathrm{TICl} 2 \mathrm{~b}-3$ were $54 \%$ $(7 / 13)$ before the introduction of protocol and 50\% (17/34) after the introduction of protocol, with no significant difference $(p=0.815$, Fisher's exact test). TICl: thrombolysis in cerebral infarction

phase, it took about 20 minutes to get from the emergency room to the MRI room and back. In the post-protocol phase, the performance of an MRI scan was omitted. Although there were some disadvantages, such as increased radiation exposure and adverse effects of contrast agents, plain CT and 3DCTA were obtained simultaneously in the CT room adjacent to the emergency room, which greatly contributed to the shorter DTN and DTP times. Third, immediately after the performance of plain CT and 3DCTA, the neurosurgeons and neurologists formulated and conveyed the treatment plan to the paramedical staff. Each of them performed their individual tasks. Fourth, the clinical engineering technologists provided device settings and intraoperative supports, which considerably reduced the NTP and PTR times. Prior to the introduction of the protocol, the emergency room nurses, radiologists, clinical engineering technologists, and emergency physicians were informed of the indications for reperfusion therapy and the importance of shortening the time. By standardizing the initial response according to the protocol, the examinations necessary for the treatment plan could be performed without delay, even in the absence of a neurosurgeon or neurologist. However, the time required for treatment at our hospital did not meet the Society of NeuroInterventional Surgery guideline recommendations. ${ }^{15)}$ After the need for reperfusion therapy has been determined, even if the paramedics act independently, the stroke team doctors must inform the family, initiate IV rt-PA, and select the appropriate device. In hospitals with limited human resources, it may be difficult to significantly reduce the time required from patient arrival to recanalization.

Although the time savings were achieved, the patient outcomes did not improve, but the number of patients with

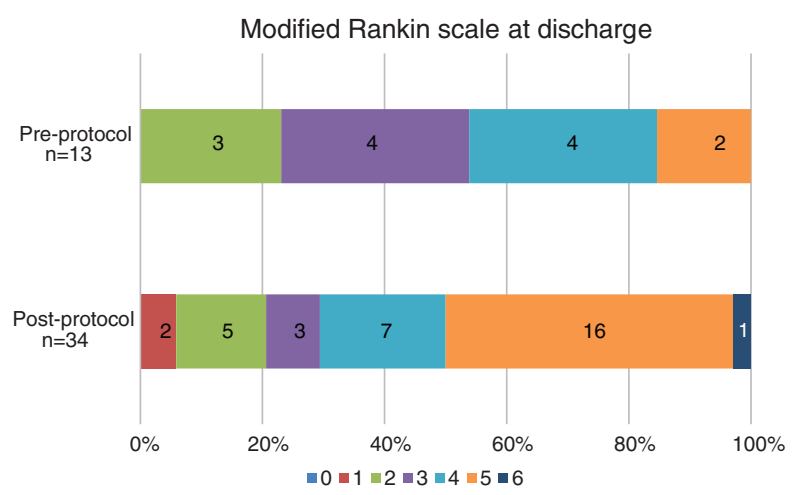

Fig. 3 The rates of good prognosis in patients with $\mathrm{mRS}$ score of $0-2$ were $23 \%(3 / 13)$ before protocol introduction and $21 \%(7 / 34)$ after protocol introduction, with no significant difference ( $p=0.854$, Fisher's exact test). The rate of poor prognosis in patients with $\mathrm{mRS}$ score $5-6$ were $15 \%(2 / 13)$ before protocol introduction and $50 \%$ $(17 / 34)$ after protocol introduction, with significant difference ( $p=$ 0.046, Fisher's exact test). mRS: modified Rankin scale

mRS score of 5-6 significantly increased (Fig. 3). This finding is likely to be caused by the low recanalization rate and revascularization of non-indicated patients due to the decision to use plain CT as an indication. Despite the advancements in the devices and the development of mechanical thrombectomy techniques in the post-protocol period, the recanalization rate at our hospital remains inadequate. Even if stent retriever and/or intra-arterial fibrinolysis have already been used in previous clinical trials, the TICI $2 \mathrm{~b}-3$ rate was still $58.7 \%-88 \%$ (Table 3). ${ }^{16-20)}$ The ADAPT technique was not inferior to the thrombectomy technique using a stent retriever. ${ }^{11)}$ Furthermore, the combined technique simultaneously use of a large-bore aspiration catheter and a stent retriever yielded a TICI $2 b-3$ rate of $>95 \% .^{21-24)}$ Although the use of either a stent retriever or aspiration catheter as a simple technique may have contributed to the poor prognosis, the inadequate recanalization rate (TICI $2 \mathrm{~b}-3$ rate: $50 \%$ ) in the post-protocol phase was due to the lack of skills of the operator. To improve the recanalization rate, a combined technique should be used as the first-line option.

Although the indications can be quickly determined by plain CT, CT-ASPECTS tends to underestimate the ischemic core compared with DWI-ASPECTS ${ }^{25)}$ and reperfusion indication cases that cannot be rescued were possibly included in post-protocol phase. Even in cases within 6 hours of onset, prognosis was better in the study using perfusion imaging and MRI to evaluate ischemic core and penumbra mismatch (Table 3). ${ }^{16-20)}$ At our hospital, 3DCTA was carried out to determine the occluded vessels and omitted the performance of an aortic dissection. However, CT perfusion (CTP) imaging was not performed 
Table 32015 RCT results and imaging criteria to decide thrombectomy

\begin{tabular}{lccl} 
& TICl $2 \mathrm{~b}-3(\%)$ & $\mathrm{mRS} 0-2(\%)$ & \multicolumn{1}{c}{ Imaging criteria } \\
MR CLEAN & 58.7 & 32.6 & CT only \\
REVASCAT & 65.7 & 43.7 & ASPECTS $\geq 7$ /ASPECTS-DWI $\geq 6$ \\
ESCAPE & 72.4 & 53 & ASPECTS $\geq 6$ and good collateral in multiphase CTA \\
EXTEND-IA & 86.2 & 71 & RAPID $^{\circledR}$ target mismatch and ischemic core $<70 \mathrm{~mL}$ \\
SWIFT PRIME & 88 & 60 & RAPID $^{\circledR}$ target mismatch and ischemic core $<50 \mathrm{~mL}$ \\
\hline
\end{tabular}

ASPECTS: Alberta Stroke Program Early CT score; DWI: diffusion-weighted imaging; mRS: modified Rankin scale; RCT: randomized controlled trial; $\mathrm{TICl}$ : thrombolysis in cerebral infarction

because of problems with the image analysis software in the emergency room. Based on the present study, we may consider updating the image analysis software to obtain CTP images. The threshold for determining the $T_{\max }$ and cerebral blood flow thresholds for determining the ischemic core by CTP depend on the elapsed time. ${ }^{26)}$ In Japan, where the RAPID system is not widely used, DWI-ASPECTS is a simple and reliable way to suppose the ischemic core. ${ }^{27)}$ It takes 20 minutes to transfer from the emergency room to the MRI room and back. In principle, the plain CT/CTA recanalization protocol was adopted except in patients with M2 obstruction and those whose disease onset was between 6 and 24 hours. However, the DWI-based recanalization protocol might be considered in patients with early presenting stroke. It is an undeniable fact that some patients with low ASPECTS show symptomatic improvement after receiving reperfusion therapy, ${ }^{28)}$ and a study has reported good results with CT-based reperfusion therapy. ${ }^{29)}$ Strict indications can be selected based on the detailed ischemic core volume and penumbral mismatch with some time consumption while wide (perhaps "over") indications can be selected with minimal examinations to achieve recanalization in the shortest time possible. However, time reduction is still an important factor, and efforts should be made to reduce the time to recanalization, taking into account the conditions of each facility and its medical area. ${ }^{6,7)}$

This study had some limitations. It was performed retrospectively, and the low effective recanalization rate may have counteracted the effect of time savings. The results of CT-based reperfusion therapy based on high effective recanalization rates, including the combined technique, are desirable.

\section{Conclusion}

The introduction of the AIS protocol has significantly reduced the time from hospital arrival to reperfusion of patients in the hyperacute phase of cerebral infarction.
A direct call to the neurologist and neurosurgeon on duty before the arrival of emergency medical service, omission of the MRI scan, and standardization of treatment protocols had a major impact on shortening the DTR. The introduction of the CT-based reperfusion protocol did not increase the number of patients with a good prognosis at discharge, but significantly increased the number of patients with poor outcomes (mRS score: 5-6). Judging the indication based on the findings on plain CT and 3DCTA was highly likely to lead to reperfusion indications in cases that cannot be rescued. In local community hospitals with a limited number of cases and human resources, the indications for reperfusion therapy should be determined based on the perfusion imaging findings or DWI-ASPECTS.

\section{Acknowledgment}

We would like to thank Dr. Hirotoshi Imamura for providing the data for the Mt. Fuji workshop on CVD.

\section{Disclosure Statement}

The authors declare that they have no conflict of interest.

\section{References}

1) The Japanese Stroke Society: Guidelines for appropriate use of intravenous thrombolysis (rt-PA), Edition 3. March 2019. https://www.jsts.gr.jp/img/rt-PA03.pdf (in Japanese).

2) Thomalla G, Simonsen CZ, Boutitie F, et al: MRI-guided thrombolysis for stroke with unknown time of onset. N Engl J Med 2018; 379: 611-622.

3) Albers GW, Marks MP, Kemp S, et al: Thrombectomy for stroke at 6 to 16 hours with selection by perfusion imaging. N Engl J Med 2018; 378: 708-718.

4) Nogueira RG, Jadhav AP, Haussen DC, et al: Thrombectomy 6 to 24 hours after stroke with a mismatch between deficit and infarct. N Engl J Med 2018; 378: 11-21. 
Mano $Y$, et al.

5) The Japan Stroke Society, The Japan Neurosurgical Society, and The Japanese Society for Neuroendovascular Therapy. Guidelines for Proper Use of Percutaneous Transluminal Cerebral Thrombus Retrieval Devices, Edition 4. March 2020. (https://www.jsts.gr.jp/img/noukessen_4.pdf) (in Japanese).

6) Emberson J, Lees KR, Lyden P, et al: Effect of treatment delay, age, and stroke severity on the effects of intravenous thrombolysis with alteplase for acute ischaemic stroke: a meta-analysis of individual patient data from randomised trials. Lancet 2014; 384: 1929-1935.

7) Saver JL, Goyal M, Van Der Lugt A, et al: Time to treatment with endovascular thrombectomy and outcomes from ischemic stroke: a meta-analysis. JAMA 2016; 316: 12791288.

8) Froehler MT, Saver JL, Zaidat OO, et al: Interhospital transfer before thrombectomy is associated with delayed treatment and worse outcome in the STRATIS registry (systematic evaluation of patients treated with neurothrombectomy devices for acute ischemic stroke). Circulation 2017; 136: 2311-2321.

9) Venema E, Groot AE, Lingsma HF, et al: Effect of interhospital transfer on endovascular treatment for acute ischemic stroke. Stroke 2019; 50: 923-930.

10) Kothari RU, Pancioli A, Liu T, et al: Cincinnati prehospital stroke scale: reproducibility and validity. Ann Emerg Med 1999; 33: 373-378.

11) Turk AS, Spiotta A, Frei D, et al: Initial clinical experience with the ADAPT technique: a direct aspiration first pass technique for stroke thrombectomy. J Neurointerv Surg 2014; 6: 231-237.

12) Tomsick T, Broderick J, Carrozella J, et al: Revascularization results in the interventional management of stroke II trial. AJNR Am J Neuroradiol 2008; 29: 582-587.

13) van Swieten JC, Koudstaal PJ, Visser MC, et al: Interobserver agreement for the assessment of handicap in stroke patients. Stroke 1988; 19: 604-607.

14) Goyal M, Menon BK, van Zwam WH, et al: Endovascular thrombectomy after large-vessel ischaemic stroke: a meta-analysis of individual patient data from five randomised trials. Lancet 2016; 387: 1723-1731.

15) McTaggart RA, Ansari SA, Goyal M, et al: Initial hospital management of patients with emergent large vessel occlusion (ELVO): report of the standards and guidelines committee of the Society of NeuroInterventional Surgery. J Neurointerv Surg 2017; 9: 316-323.

16) Saver JL, Goyal M, Bonafe A, et al: Stent-retriever thrombectomy after intravenous t-PA vs. t-PA alone in stroke. N Engl J Med 2015; 372: 2285-2295.
17) Jovin TG, Chamorro A, Cobo E, et al: Thrombectomy within 8 hours after symptom onset in ischemic stroke. N Engl J Med 2015; 372: 2296-2306.

18) Goyal M, Demchuk AM, Menon BK, et al: Randomized assessment of rapid endovascular treatment of ischemic stroke. N Engl J Med 2015; 372: 1019-1030.

19) Campbell BC, Mitchell PJ, Kleinig TJ, et al: Endovascular therapy for ischemic stroke with perfusion-imaging selection. N Engl J Med 2015; 372: 1009-1018.

20) Berkhemer OA, Fransen PS, Beumer D, et al: A randomized trial of intraarterial treatment for acute ischemic stroke. N Engl J Med 2015; 372: 11-20.

21) Goto $S$, Ohshima $T$, Ishikawa $K$, et al: A stent-retrieving into an aspiration catheter with proximal balloon (ASAP) technique: a technique of mechanical thrombectomy. World Neurosurg 2018; 109: e468-e475.

22) Maus V, Behme D, Kabbasch C, et al: Maximizing firstpass complete reperfusion with SAVE. Clin Neuroradiol 2018; 28: 327-338.

23) McTaggart RA, Tung EL, Yaghi S, et al: Continuous aspiration prior to intracranial vascular embolectomy (CAPTIVE): a technique which improves outcomes. J Neurointerv Surg 2017; 9: 1154-1159.

24) Massari F, Henninger N, Lozano JD, et al: ARTS (Aspiration-retriever technique for stroke): initial clinical experience. Interv Neuroradiol 2016; 22: 325-332.

25) Nezu T, Koga M, Nakagawara J, et al: Early ischemic change on CT versus diffusion-weighted imaging for patients with stroke receiving intravenous recombinant tissue-type plasminogen activator therapy: stroke acute management with urgent risk-factor assessment and improvement (SAMURAI) rt-PA registry. Stroke 2011; 42: 2196-2200.

26) d'Esterre CD, Boesen ME, Ahn SH, et al: Time-dependent computed tomographic perfusion thresholds for patients with acute ischemic stroke. Stroke 2015; 46: 3390-3397.

27) Yoshimoto $T$, Inoue $M$, Yamagami $H$, et al: Use of diffusion-weighted imaging-alberta stroke program early computed tomography score (DWI-ASPECTS) and ischemic core volume to determine the malignant profile in acute stroke. J Am Heart Assoc 2019; 8: e012558.

28) Kakita H, Yoshimura S, Uchida K, et al: Impact of endovascular therapy in patients with large ischemic core: subanalysis of recovery by endovascular salvage for cerebral ultra-acute embolism Japan registry 2. Stroke 2019; 50: 901-908.

29) The committee of Mt. Fuji Workshop on CVD: The 34th meeting of The Mt. Fuji Workshop on CVD. NEURON Publishing, Tokyo, 2016, 8-13. (in Japanese). 